\title{
Ages of asteroid families estimated using the YORP-eye method
}

\author{
Paolo Paolicchi ${ }^{\oplus},{ }^{1 \star}$ F. Spoto, ${ }^{2 \star}$ Z. Knežević ${ }^{3 \star}$ and A. Milani ${ }^{4} \dagger$ \\ ${ }^{1}$ Department of Physics, University of Pisa, Largo Pontecorvo 3, I-56127 Pisa, Italy \\ ${ }^{2}$ IMMCE, Observatoire de Paris, Av. Denfert-Rochereau 77, F-75014 Paris, France \\ ${ }^{3}$ Serbian Academy of Sciences and Arts, Kneza Mihaila 35, 11000 Belgrade, Serbia \\ ${ }^{4}$ Department of Mathematics, University of Pisa, Largo Pontecorvo 5, I-56127 Pisa, Italy
}

Accepted 2018 December 14. Received 2018 December 13; in original form 2018 October 18

\begin{abstract}
Recently, we have shown that it is possible, despite several biases and uncertainties, to find footprints of the Yarkovsky-O'Keefe-Radzievskii-Paddack (YORP) effect, concerning the members of asteroid dynamical families, in a plot of the proper semimajor axis versus magnitude (the so-called V-plot). In our previous work, we introduced the concept of the YORP-eye, the depopulated region in the V-plot, whose location can be used to diagnose the age of the family. In this present paper, we complete the analysis using an improved algorithm and an extended data base of families, and we discuss the potential errors arising from uncertainties and from the dispersion of the astronomical data. We confirm that the analysis connected to the search for the YORP-eye can lead to an estimate of the age, which is similar and strongly correlated to that obtained by the analysis of the V-slope size-dependent spreading due to the Yarkovsky effect. In principle, the YORP-eye analysis alone can lead to an estimate of the ages of other families, which have no independent age estimates. However, these estimates are usually affected by large uncertainties and, often, are not unique. Thus, they require a case-by-case analysis to be accepted, even as a rough first estimate.
\end{abstract}

Key words: minor planets, asteroids: general.

\section{INTRODUCTION}

In a recent paper, Paolicchi \& Knežević (2016, hereafter Paper I) extensively discussed the possibility of detecting footprints of the Yarkovsky-O'Keefe-Radzievskii-Paddack (YORP) effect in asteroid dynamical families by analysing the distribution of its members in the so-called V-plot (i.e. the absolute magnitude $H$ or inverse size $1 / D$ versus proper semimajor axis $a$ ). As is well known, the YORP effect often causes the migration of the spin vector pole towards extreme obliquities measured from the normal to the orbital plane (Bottke et al. 2002, 2006; Vokrouhlický \& Čapek 2002; Micheli \& Paolicchi 2008; Nesvorný \& Vokrouhlický 2008; Vokrouhlický et al. 2015). In dynamical families, this process has to be combined with the migration in the semimajor axis due to the diurnal Yarkovsky effect (Farinella, Vokrouhlický \& Hartmann 1998; Farinella \& Vokrouhlický 1999; Bottke et al. 2002; Chesley et al. 2003; Vokrouhlický et al. 2015), which is faster (for a given size of the body) for extremely oblique rotation axes. The clustering of axes causes a clustering in $a$ close to the borders of the V-plot of several families (Vokrouhlický et al. 2006; Bottke et al. 2015).

^E-mail: paolo.paolicchi@unipi.it (PP); federica.spoto@oca.eu (FS); zoran@aob.rs (ZK)

$\dagger$ Deceased
However, this effect is not easily detected for other families (Spoto, Milani \& Knežević 2015).

In Paper I, we introduced the so-called central depletion parameter $R(H)$ as a function of the absolute magnitude, and we discussed how the maxima of $R(H)$ can provide information about the age of the family. We assumed that a maximum depletion is present at a given $H$ whenever the duration of a YORP cycle, for that value of $H$, is equal to - or proportional to, with a fixed constant of proportionality - the age of the family. This assumption led to the definition of the YORP-eye. In Paper I, we introduced an adimensional parameter, the YORP-age (represented in the equations as $Y_{\text {age }}$; see equation 6). Assuming $f(A)=1$ (see Paper I for a discussion), $Y_{\text {age }}$ is simply given by

$Y_{\text {age }}=\tau_{\mathrm{f}} A / a^{2}$,

where $\tau_{\mathrm{f}}$ is the age of the family (in Myr), $A$ is the albedo and $a$ is the semimajor axis (in au).

If we know the age and the other average properties of the family, we are able to compute $Y_{\text {age }}$ and, in turn, to estimate at what value of $H$ the maximum of the function $R(H)$ is expected. The main purpose of Paper I was to compare the expected and computed maxima, and the main result was that these are often not too different, and fairly well correlated.

In this paper, the analysis attempted in Paper I is improved, extended and carried to more quantitative and detailed conclusions. 
The analysis is extended to 48 families for which Milani et al. (2016, 2017) estimated the age. The estimate was based on the spreading of the V-plot with time, as a consequence of the Yarkovsky effect. These families are hereafter referred to as Yarkaged families. This sample is larger compared with the sample used in Paper I, both because of the increased list of Yarkaged families and because of the possibility, allowed by the new version of the algorithm, of performing a reliable analysis of smaller families (down to 100 members, compared with 250 members in the previous work). As in Paper I, we performed this analysis on the basis of the $a-H \mathrm{~V}$ shaped plots of the various families, using a sample of about 130000 family members, classified according to the method discussed in Milani et al. (2014), within a general list exceeding 500000 objects. In our list, we also include a few families, again with the number of members exceeding the minimum value, for which no previous age estimate exists (hereafter referred to as un-Yarkaged families).

\section{NEW DATA, NEW ALGORITHM AND AN IMPROVED ANALYSIS}

In this work, we have analysed a total of 64 families with more than 100 members, obtained from the current version of the Astdys data base (Knežević \& Milani 2003). Using the method discussed in Milani et al. (2014), for 36 of the families, a twofold age estimate has been provided, computed from the slope of the left (or IN) and right (or OUT) wings of the V-plot; usually, the two values are not exactly equal, and in a few cases they are even significantly different. However, for 33 families, it has been possible to reconcile the two estimates, which are consistent within an error bar (Milani et al. 2017). For the remaining three families, the two ages are definitely inconsistent with each other. These families might have a peculiar collisional history; the two ages might correspond to different events. For instance, in the case of the family of (4) Vesta the fragments have presumably been created by two (or more) cratering collisions. For 12 'one-sided' families only one age estimate has been possible. Again, this might be the consequence of a complex collisional history and also of dynamical processes: the family might be asymmetrical due to the cutting effect of a strong resonance. The analysis of individual cases has been presented in separate papers. Finally, the remaining 16 families have no age estimate obtained with the method based on the Yarkovsky effect (un-Yarkaged families), even if, for some of them, other age estimates are available in the literature. The main properties of the families in our sample are detailed in Tables 1, 2 and 3.

The analysis of the updated set of families has been improved with a small but relevant change in the algorithm. In Paper I, we searched for the maxima of the depletion parameter $R(H)$ with a running box method, with a fixed box size of 100 bodies. The choice was conservative, excluding the possibility of analysing small families, and also potentially masking significant features, especially in the low- $H$ tail, where a jump by 100 bodies might mean to pass in a single step from the largest bodies to, by far, smaller bodies. The main purpose of Paper I, however, was to show that the YORP-eye search is a sensible and useful concept, and that the results can provide hints about the age of the family. From this point of view, such a conservative and cautious approach was reasonable.

In this present paper, we are instead interested in obtaining significant information about the age and in comparing it with other family age data. We are also interested in introducing the possibility of using the YORP-eye method alone to obtain a first guess for the age independently from the other results possibly present in the literature. In order to improve our potential for analysis, we have
Table 1. Summary of the data for the families used in the computations. The table lists families with two consistent estimates of Yarkage. For each family (Corfam $=$ the label of the core family; see Spoto et al. 2015), we list the number of members (Mem). We then give the average value of the geometrical albedo (AveAl), and its dispersion in the family (Alvar). Next, we give the average proper semimajor axis, Sma, of the family (in au). Finally, we present the age (in Myr) computed according to the method of Milani et al. (2014) and the estimated error (Ager). The updated estimate of the error is computed according to the method discussed by Milani et al. (2017). Note also the following: the family 163 is a combination of the nominal family 163 with the family 5026; we list the family whose largest member is the asteroid 1521 as 293 ; we list the family whose largest member is the asteroid 363 (Padua) as 110; we list the family whose largest member is the asteroid 686 (Gersuind) as 194 . Note also that the family 18405 was already called as Brixia (521 Brixia is now considered to be a background object) and we take the nominal family 31 , in spite of the problems discussed in the text. Regarding the family 9506, see the discussion in Section 4.7.

\begin{tabular}{|c|c|c|c|c|c|c|}
\hline Corfam & Mem & AveAl & Alvar & Sma & Age & Ager \\
\hline 3 Juno & 1693 & 0.253 & 0.060 & 2.670 & 463 & 110 \\
\hline 5 Astraea & 6169 & 0.269 & 0.080 & 2.580 & 328 & 71 \\
\hline 10 Hygiea & 3147 & 0.073 & 0.020 & 3.160 & 1347 & 220 \\
\hline 20 Massalia & 7820 & 0.249 & 0.070 & 2.400 & 180 & 27 \\
\hline 24 Themis & 5612 & 0.069 & 0.020 & 3.150 & 3024 & 632 \\
\hline 31 Euphrosyne & 1384 & 0.061 & 0.020 & 3.150 & 1225 & 304 \\
\hline 110 Lydia & 899 & 0.171 & 0.040 & 2.740 & 238 & 40 \\
\hline 158 Koronis & 7390 & 0.240 & 0.060 & 2.890 & 1746 & 296 \\
\hline 163 Erigone & 1023 & 0.055 & 0.010 & 2.370 & 224 & 36 \\
\hline 194 Prokne & 379 & 0.150 & 0.040 & 2.590 & 1448 & 348 \\
\hline 221 Eos & 16040 & 0.157 & 0.050 & 3.040 & 1466 & 216 \\
\hline 293 Brasilia & 845 & 0.174 & 0.040 & 2.850 & 143 & 56 \\
\hline 302 Clarissa & 236 & 0.053 & 0.020 & 2.400 & 50 & 10 \\
\hline 396 Aeolia & 529 & 0.106 & 0.030 & 2.740 & 95 & 21 \\
\hline 434 Hungaria & 1869 & 0.380 & 0.100 & 1.940 & 206 & 45 \\
\hline 480 Hansa & 1164 & 0.286 & 0.070 & 2.630 & 895 & 164 \\
\hline 569 Misa & 647 & 0.058 & 0.020 & 2.650 & 259 & 95 \\
\hline 606 Brangane & 325 & 0.121 & 0.030 & 2.580 & 46 & 8 \\
\hline 668 Dora & 1742 & 0.058 & 0.010 & 2.780 & 506 & 116 \\
\hline 808 Merxia & 1263 & 0.248 & 0.060 & 2.750 & 329 & 50 \\
\hline 845 Naema & 375 & 0.065 & 0.010 & 2.940 & 156 & 23 \\
\hline 847 Agnia & 3336 & 0.242 & 0.060 & 2.780 & 753 & 151 \\
\hline 1040 Klumpkea & 1815 & 0.204 & 0.100 & 3.130 & 663 & 154 \\
\hline 1128 Astrid & 548 & 0.052 & 0.010 & 2.780 & 150 & 23 \\
\hline 1303 Luthera & 232 & 0.052 & 0.010 & 3.220 & 276 & 62 \\
\hline 1547 Nele & 344 & 0.355 & 0.070 & 2.640 & 14 & 4 \\
\hline 1726 Hoffmeister & 2095 & 0.048 & 0.010 & 2.780 & 332 & 67 \\
\hline 1911 Schubart & 531 & 0.039 & 0.010 & 3.970 & 1557 & 343 \\
\hline 3330 Gantrisch & 1240 & 0.047 & 0.010 & 3.150 & 460 & 128 \\
\hline 3815 Konig & 578 & 0.051 & 0.010 & 2.570 & 51 & 10 \\
\hline 9506 Telramund & 325 & 0.245 & 0.070 & 2.990 & 219 & 49 \\
\hline 10955 Harig & 918 & 0.251 & 0.070 & 2.700 & 462 & 121 \\
\hline 18405 1993FY12 & 159 & 0.184 & 0.040 & 2.850 & 83 & 21 \\
\hline
\end{tabular}

systematically reduced the box size. According to Paolicchi et al. (2017), who analysed several different sizes, the reduction of the box size does not significantly affect the results, with the exception of some features occurring in the distribution tail containing the large bodies. Here, we have used a box size of 20 bodies for families of up to 250 members, of 30 in the range 250-2000 and of 50 for the largest families. Moreover, in a few cases, we have explored the limit case with a box size of 10 . As we discuss in the following, this choice is sometimes appropriate to improve the resolution of the $R(H)$ function, and sometimes necessary to inspect the features close to the low- $H$ region. 
Table 2. The same as in Table 1 but for families with two ages that cannot be explained with a single collisional event (first three lines) and for families with only one age estimate. A zero value is given when it has not been possible to estimate the age. Note that Age1 and Ageler refer to the left (or IN) wing, while Age2 and Age2er refer to the right (or OUT) wing. Note also that we list as family of (93) Minerva the family for which, in reality, the largest member is the asteroid 1272 Gefion.

\begin{tabular}{lcccccccc}
\hline Corfam & Mem & AveAl & Alvar & Sma & Age1 & Age1er & Age2 & Age2er \\
\hline 4 Vesta & 10612 & 0.355 & 0.10 & 2.36 & 930 & 217 & 1906 & 659 \\
15 Eunomia & 9756 & 0.260 & 0.08 & 2.62 & 1955 & 421 & 1144 & 236 \\
25 Phocaea & 1248 & 0.253 & 0.120 & 2.32 & 1187 & 319 & 0 & 0 \\
87 Sylvia & 191 & 0.059 & 0.020 & 3.52 & 0 & 0 & 1119 & 282 \\
93 Minerva & 2428 & 0.256 & 0.100 & 2.77 & 1103 & 386 & 0 & 0 \\
135 Hertha & 15984 & 0.060 & 0.020 & 2.39 & 761 & 242 & 0 & 0 \\
145 Adeona & 2069 & 0.062 & 0.010 & 2.65 & 794 & 184 & 0 & 0 \\
170 Maria & 2958 & 0.261 & 0.080 & 2.59 & 0 & 0 & 1932 & 422 \\
283 Emma & 577 & 0.049 & 0.01 & 3.05 & 290 & 67 & 628 & 234 \\
375 Ursula & 731 & 0.062 & 0.020 & 3.17 & 3483 & 1035 & 0 & 0 \\
752 Sulamitis & 193 & 0.055 & 0.010 & 2.44 & 341 & 109 & 0 & 0 \\
945 Barcelona & 346 & 0.300 & 0.100 & 2.62 & 203 & 56 & 0 & 0 \\
1658 Innes & 775 & 0.264 & 0.070 & 2.58 & 0 & 0 & 464 & 143 \\
2076 Levin & 1536 & 0.202 & 0.070 & 2.29 & 0 & 0 & 366 \\
3827 Zdenekhorsky & 1050 & 0.074 & 0.020 & 2.73 & 154 & 34 & 0 \\
\hline
\end{tabular}

Table 3. The same as in Tables 1 and 2 but for the un-Yarkaged families.

\begin{tabular}{lcccc}
\hline Corfam & Mem & AveAl & Alvar & sma \\
\hline 96 Aegle & 120 & 0.071 & 0.010 & 3.06 \\
148 Gallia & 137 & 0.268 & 0.070 & 2.76 \\
298 Baptistina & 177 & 0.210 & 0.070 & 2.27 \\
410 Chloris & 120 & 0.095 & 0.030 & 2.74 \\
490 Veritas & 2139 & 0.070 & 0.020 & 3.17 \\
778 Theobalda & 574 & 0.066 & 0.020 & 3.18 \\
883 Matterania & 170 & 0.274 & 0.100 & 2.24 \\
1118 Hanskya & 116 & 0.057 & 0.010 & 3.20 \\
1222 Tina & 107 & 0.160 & 0.030 & 2.79 \\
1298 Nocturna & 186 & 0.073 & 0.030 & 3.15 \\
1338 Duponta & 133 & 0.279 & 0.100 & 2.28 \\
2782 Leonidas & 111 & 0.065 & 0.010 & 2.68 \\
12739 1992DY7 & 298 & 0.186 & 0.100 & 2.72 \\
13314 1998RH71 & 241 & 0.051 & 0.010 & 2.78 \\
18466 1995SU37 & 257 & 0.240 & 0.080 & 2.78 \\
31811 1999NA41 & 144 & 0.126 & 0.040 & 3.11 \\
\hline
\end{tabular}

We have also introduced a further improvement, in order to avoid the occurrence of meaningless unphysical maxima. While the large members of a family are usually well below the observational completeness limit, and thus they are fully representative of the mass/size distribution within the family (apart from the presence of potential interlopers, not always easily identified), the high- $H$ tail is severely biased. In general, we expect a distribution $\mathrm{d} n / \mathrm{d} H$ monotonically increasing with $H$, at least within a significant range. Thus, a decrease indicates observational selection effects (perhaps combined with evolutionary effects; Morbidelli et al. 2003). In our analysis, we have truncated our search of maxima of $R(H)$ at $H$ values for which the distribution $\mathrm{d} n / \mathrm{d} H$ has already significantly begun to decrease. Typically, the resulting cut-off is at $H=16 / 17$ mag, which is, by the way, usually not critical for our search of the YORP-eye. We analyse this point in detail in the following.

Here, we wish to discuss another significant aspect, concerning the resulting plots of the depletion parameter.

The plots we used previously in Paper I were showing only the maxima of the function $R(H)$, that is, we have kept only the values $R M A X(H)$, which are local maxima. However, the maxima are often very numerous, and also the function $\operatorname{RMAX}(H)$ exhibits, in some cases, sawtooth-like features or high-frequency fluctuations. Thus, we have identified the significant maxima, looking at the plot of $R M A X(H)$ and searching for maxima of the maxima. When several significant maxima are present within a small $H$ range, we select only the one with the highest value of $R$. In this way, we have selected a number $N_{\max }$ of significant maxima, which is usually 2 (the most frequent case) or 3, with a few cases with only one maximum and one case for which $N_{\max }=4$.

From the point of view of the theory, we know the following.

(i) The original properties of the family might (or might not) entail one - or even more than one - maximum $R$, which is usually masked by the subsequent evolution, but which can survive if it involves the large bodies (whose orbital elements are not affected, or are only moderately affected, by the Yarkovsky-driven mobility).

(ii) We have no idea, at the moment, of what happens after several YORP cycles. Perhaps the central depletion is partially or totally masked (which has been our starting assumption when searching for the YORP-eye) but it is also possible that other significantly depleted regions appear.

(iii) For the reasons above, the YORP-eye due to YORP/Yarkovsky effects should correspond to a significant maximum of $R$ located at the smallest or at the second smallest value of $H$ (and, in exceptional cases, to the third; see the later discussion concerning the family of (221) Eos). We have systematically followed this guideline, to choose among multiple significant maxima.

(iv) We have no way to distinguish theoretically between the candidate maxima, as defined above, so, whenever possible, we have chosen one providing a better fit with the Yarkovsky estimated age. The physical processes affecting the original properties and the evolution of the V-plot with time are extremely complex. Among others, the presence of resonances can open gaps that can mix with those due to the YORP effect, or even mask them. Thus, the ability to detect footprints, also in such a fuzzy context, is a significant success.

(v) In some cases, the possible maxima do not suggest any reasonable agreement (also taking into account the errors) with the Yarkovsky ages. As we discuss in the following, these cases (which we call OUTRANGE cases) can be easily explained in 


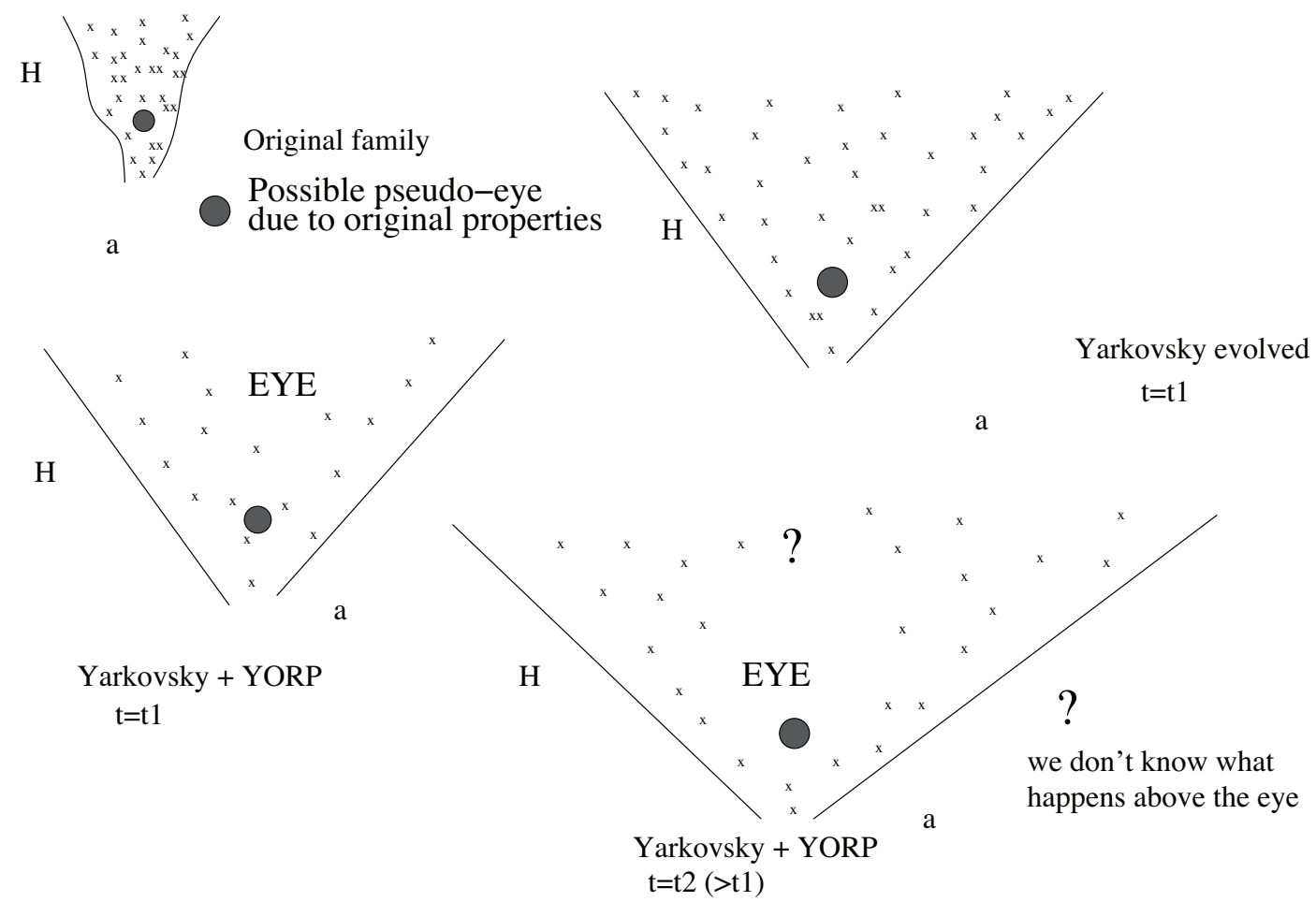

Figure 1. A schematic diagram of the expected appearance of the regions in the $a-H$ plane occupied by the members of a family ( $a$ is in abscissa and $H$ is in ordinate). The top-left diagram refers to the initial structure of the family, as originated in the family-forming collision, and due to the individual properties of the colliding bodies, to the impact geometry, etc.; the shape is not clearly defined, even if a slight general trend towards a larger spread in $a$ for the smaller members can be expected. In this diagram, a false YORP-eye (dark circle) can appear, due to the original distribution of orbital elements. If this false eye is in the region of large objects, which evolve slower, it can be preserved also at later times. The other diagrams represent the evolved family, taking into account Yarkovsky and YORP effects, with the expected formation of the true YORP-eye, and the uncertainties concerning what happens at later times, after several YORP cycles. We recall that the eye shifts with time towards the region of brighter objects.

terms of an elementary statistical argument. In other cases, we have only one-wing Yarkovsky age definition, or two values that are very different. It is necessary to discuss these case-by-case. Finally, we have families without any previous age estimate. We can use the values obtained from the YORP-eye method but, unfortunately, we have to keep all the estimates based on the various candidate maxima.

The problem of multiple maxima is explained by Fig. 1.

\section{GOOD AND BAD CASES}

For several families, it is straightforward to analyse $R M A X(H)$ and to compare the significant maxima with the values expected according to the available age estimates. In these cases, the two ages estimated by the slope of the two wings in the V-plot, according to Yarkovsky effect, are nearly equal. Among the 36 families with two age estimates, in 33 cases the two values can be considered as consistent, and a best-fitting value, together with an error bar, can be computed. If the most significant maximum of the depletion parameter is sharp and close to the value(s) expected according to the estimated ages, we definitely have a 'good' case, as happens, for instance, for the family of (845) Naema. In the top panel of Fig. 2, we show the $R M A X(H)$ function, compared with the expected values, while we include, for comparison, the V-plot of the same family (Fig. 2, bottom panel). Note that here and in the following R-plots, we compare the $R M A X(H)$ function with the maxima that are expected from the Yarkovsky-driven slope of the V-plot, not taking into account the possible offset due to the calibration (see also the discussion in Paper I). However, we can anticipate that, as a result of the present improved and extended computations, the offset will be small, with relevant consequences only for the age estimate of very young families. In fact, as we discuss in the next section, the age computed according to YORP-eye method, and with the standard calibration (defined in Paper I), has to be decreased by less than 20 per cent. This calibration entails a change in the relevant $H$ by about $0.15 \mathrm{mag}$, a small correction not affecting the present discussion. However, the best-fitting analysis reveals the presence of an additional constant term in the calibration, of the order of $50 \mathrm{Myr}$, which can be relevant for the young families.

The case of family (221) Eos is a little more troublesome. The $\operatorname{RMAX}(H)$ plot (Fig. 3, top panel) exhibits two very sharp maxima around $11 \mathrm{mag}$, and one of them is even above the upper limit of the figure. However, as it is easy to see from Fig. 3 (bottom panel), in the corresponding magnitude region, the family is extremely asymmetric, and these maxima are presumably dependent on the original properties of the family itself. Thus, in this one very peculiar case, the third (in order of increasing $H$ ) significant maximum can be taken into account. Note that it provides a very good fit with the expected values. However, as can be seen by inspection of Fig. 3 (bottom panel), the original properties of the family also play a role for this feature.

Different problems concern the dynamical families with two unconsistent age estimates. These two estimates might be related to a complex collisional history. For instance, the two different slopes of the wings of the 4 Vesta family might be correlated with two past 
FAMILY 845 Naema $\operatorname{RMAX}(\mathrm{H})$

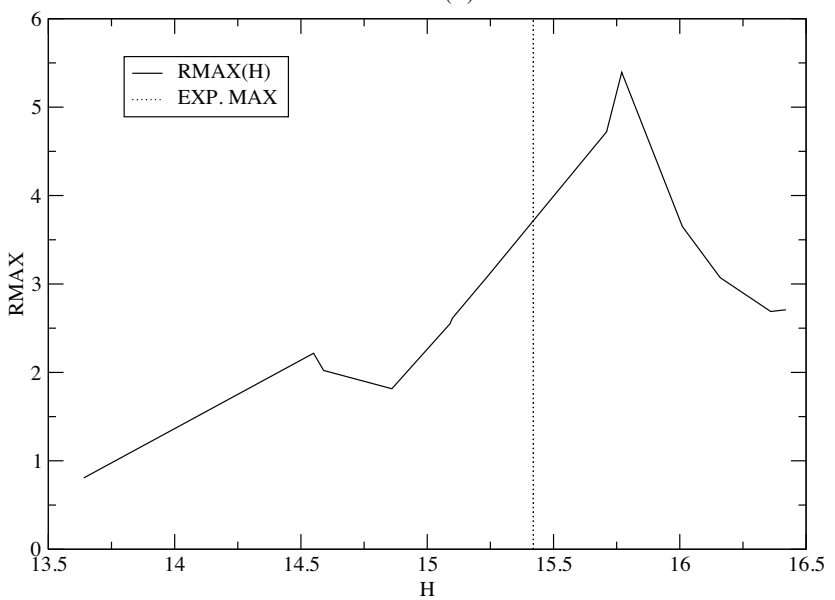

FAMILY 845 Naema

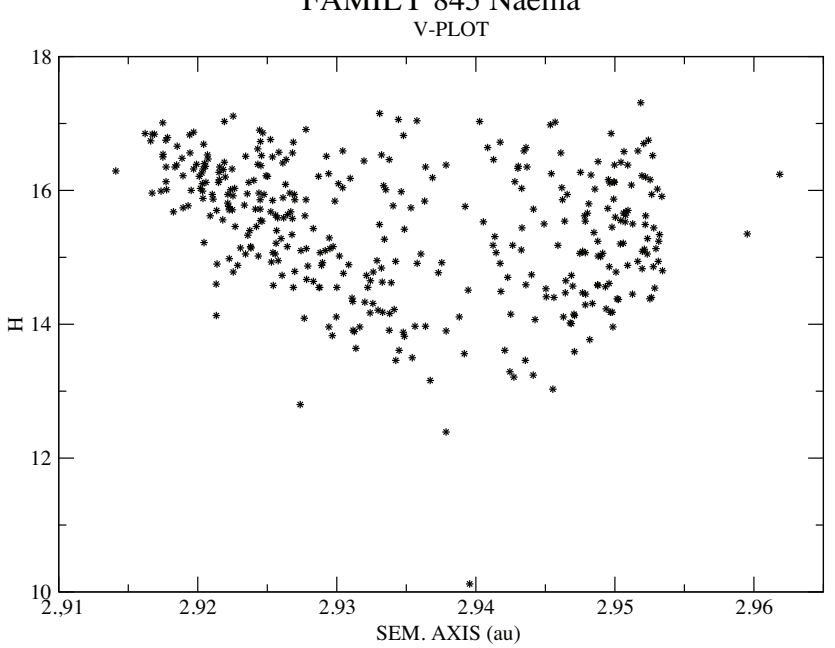

Figure 2. Top panel: the $R M A X(H)$ function for family 845 , compared with the expected value, which is in fair agreement with the computed maximum. Bottom panel: V-plot of family 845. The central depletion is apparent, even if the identification of the maximum depletion at $H \simeq 16 \mathrm{mag}$ is not obvious.

major collisional events, witnessed by the two big craters featuring on the surface of the asteroid (Rheasilvia and Veneneia). Thus, every family is, in principle, a unique case, and we can also expect different outcomes from our YORP-based analysis. For instance, for the family of (283) Emma, the comparison of $R M A X(H)$ with the two expected maxima shows a potentially significant maximum (unfortunately not very sharp) close to the OUT expected values, as shown in Fig. 4. Note that, as discussed in Paper I, maxima for which $R<1$ can also be significant. Regarding the IN expected maximum, we find either a poor fit with the absolute maximum of $R M A X(H)$ or a very good fit with another weakly significant maximum. In these cases, the comparison is less significant, entailing arbitrary choices.

The problem of asymmetric families, especially those for which only one wing provides an estimate of the age, is very complex. The causes of the asymmetry are different and not always unequivocal. We have discussed in Paolicchi et al. (2017) a few cases for which the asymmetry is mainly due to the presence of neighbouring resonances. In these cases, a possible improvement of the analysis can be achieved by a mirroring procedure. However, as the improvements
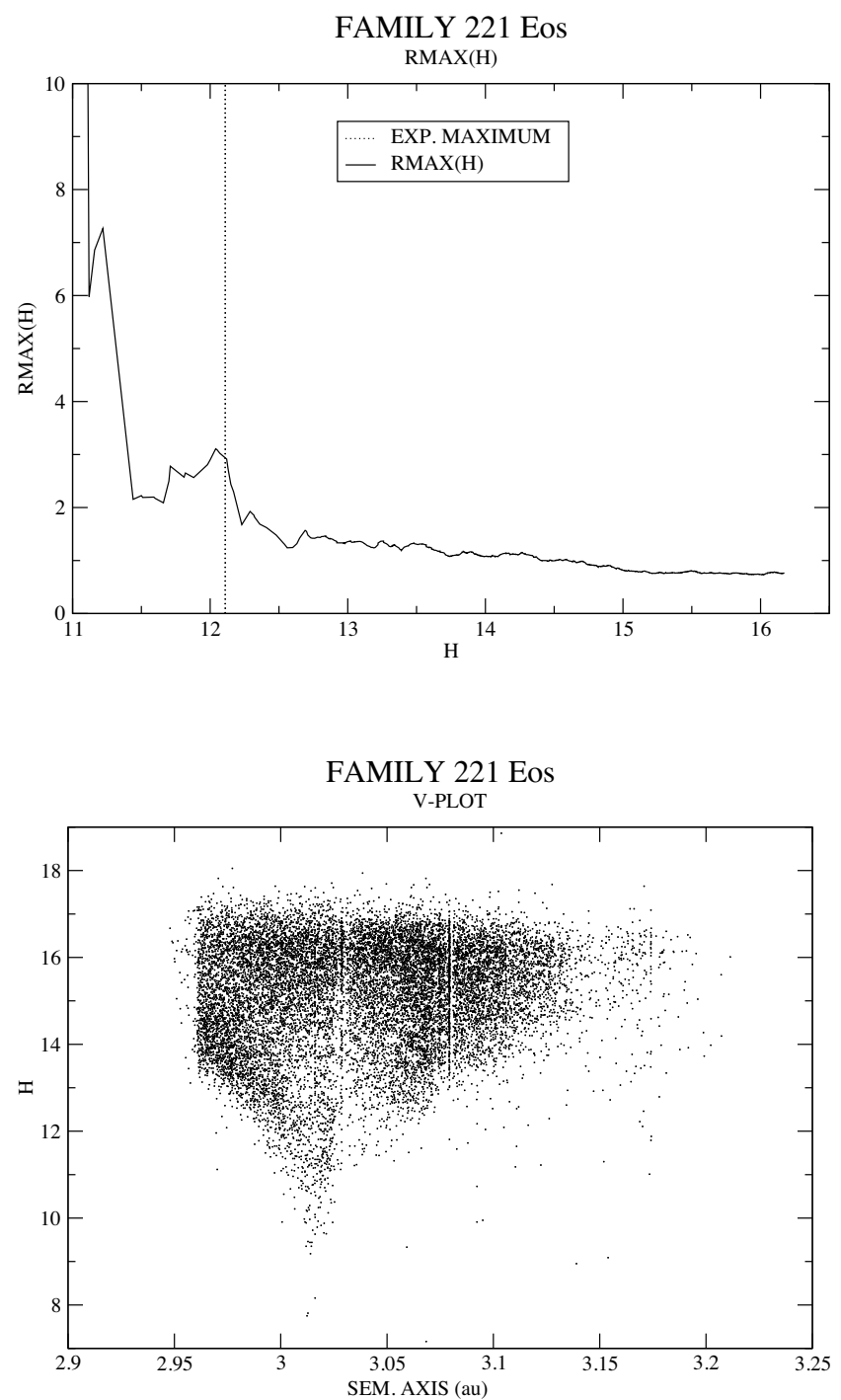

Figure 3. Top panel: the $R M A X(H)$ function for family 221 , compared with the expected value, which is in good agreement with the obtained significant maximum, although not the one corresponding to the highest value. Bottom panel: the V-plot of family 221 . The left-right asymmetry seems to dominate the overall structure of the family, at least for bright objects.

are not always very significant, and as the uncritical extension of the mirroring procedure to all the asymmetric families would be wrong, we have used for our analysis, in the case of single-aged families, the nominal family and the available age as the benchmark for comparison.

Finally, we have several cases for which it is difficult to obtain an acceptable fit between the YORP and Yarkovsky ages, even taking into account the errors. For most cases, the reason is simple: the expected maxima are in the range of $H$ for which we do not have enough bodies for a sensible statistical analysis. Often, but not always, the families involved are old, and thus the expected YORP eye is in a region with very few large bodies. In several cases, it is possible to solve the problem, and to find a significant maximum, repeating the computation with a reduced box size of 10 . The resulting $R M A X(H)$ curve is obviously more noisy. Note also that the value of $R M A X$ computed with a reduced box size is usually larger. The explanation is trivial: the range in $a$, which is used to define the seven $a$ bins (see Paper I for a detailed discussion) in the 


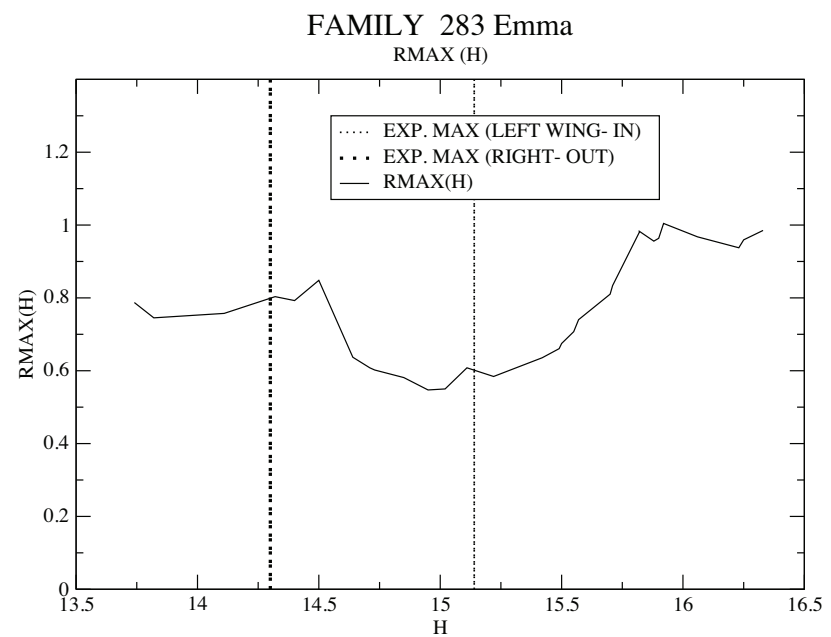

Figure 4. The $\operatorname{RMAX}(H)$ function for family 283, compared with the expected values (left and right wings); these are very different, but both are not far from local maxima of the curve.

FAMILY 163 Erigone (+5026 Martes)

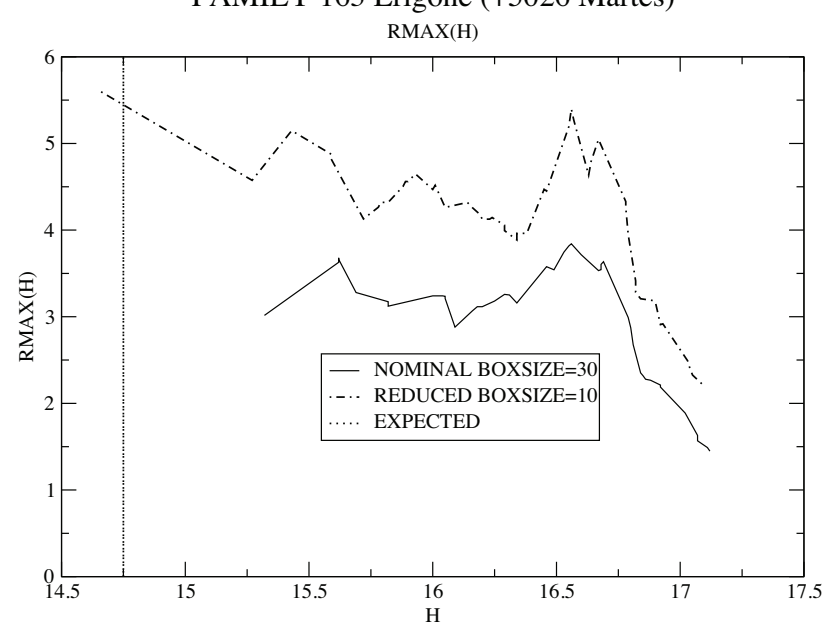

Figure 5. The $R M A X(H)$ function for family 163 , compared with the expected values (left and right wings); RMAX is also computed with a reduced box size.

box corresponding to a given value of $H$, is fixed by the extreme $a$ values in the box. With this definition, the extreme bins (1 and 7) both gain one body from scratch. This bias is negligible for large values of the box size, not so when we have a total of 10 bodies in the box.

The case of the family of (163) Erigone (combined, as in Paper I, with the family of (5026) Martes) is representative of families that can be solved with a reduced box size. As shown by Fig. 5, it is possible to find a maximum very close to the expected values only with the choice of a reduced box size. With the normal box size, the expected values are simply out of the domain of the function $R M A X(H)$.

For other families, it is simply impossible, even when reducing the value of the box size, to obtain maximum values of the function for $H$ corresponding to the expected values. For reasons that will become more clear in the following, we have discarded cases for which we do not find a good fit of Yarkovsky/YORP ages and for which, because we take as the significant maximum the lowest $H$
Table 4. The OUTRANGE families (see text). For these families, the Yarkage (in Myr) obtained with the analysis based on the Yarkovsky effect significantly exceeds the maximum age (Max $Y_{\text {age }}$ ) which would be obtained by the YORP method were the significant maximum located exactly at the low- $H$ edge. We recall that family 194 is now currently defined as 686 Gersuind. Note that the family 4 has been reported twice, using the two inconsistent Yarkages. Note also that the family (1658) Innes exhibits a severe asymmetry due to dynamical reasons, and might be corrected with the mirroring procedure described in Paolicchi et al. (2017).

\begin{tabular}{lccc}
\hline Corfam & Yarkage & Minimum $\mathrm{H}$ & Max Yage \\
\hline 3 Juno & 463 & 14.11 & 110 \\
4 Vesta & 930 & 13.03 & 171 \\
4 Vesta & 1906 & 13.03 & 171 \\
20 Massalia & 180 & 14.84 & 46 \\
194 Prokne & 1448 & 14.01 & 195 \\
434 Hungaria & 206 & 14.31 & 33 \\
480 Hansa & 895 & 12.83 & 310 \\
1658 Innes & 464 & 13.67 & 97 \\
2076 Levin & 366 & 13.77 & 140 \\
10955 Harig & 462 & 14.76 & 63 \\
\hline
\end{tabular}

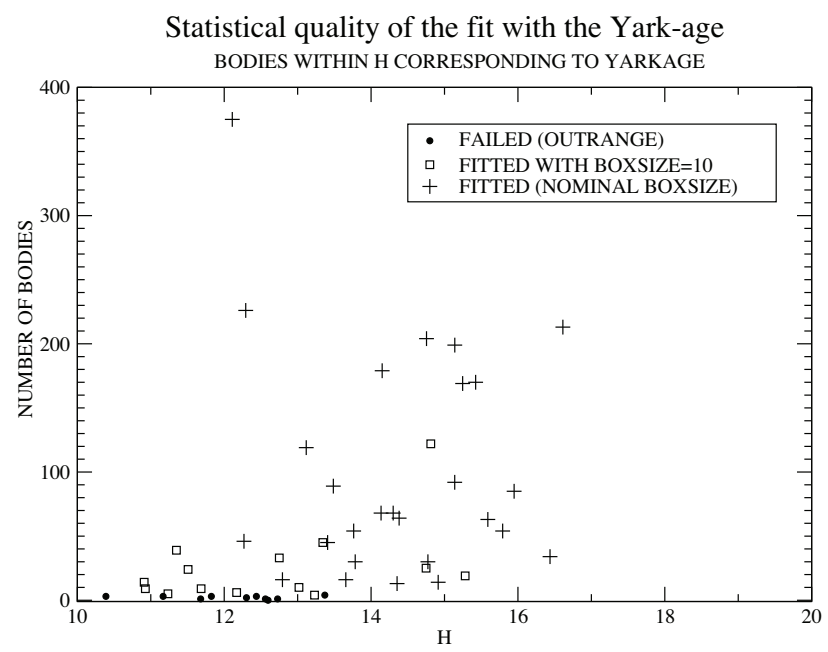

Figure 6. The number of bodies brighter than the $H$ value corresponding to the expected maximum of $R M A X(H)$, according to the estimated Yarkovsky ages (nominal values). The families for which the fit has been impossible are represented as filled circles, those for which the fit has been done with a reduced box size are represented with squares, while those for which the fit has been performed with the nominal box size are represented by crosses.

value in $\operatorname{RMAX}(H)$, we obtain a YORP-age lower than half the Yarkovsky age. Note that the criterion of factor of 2 in age (see the discussion below) corresponds essentially to a difference of about 0.75 mag in the magnitudes between the expected and the computed maxima. The criterion to reject the fit is more severe than that used in Paper I, and consequently the number of OUTRANGE families is larger. The list of these OUTRANGE families is reported in Table 4.

The problems concerning the identification of a good fit for several families can be clarified by Fig. 6 . As shown by the figure, for all the OUTRANGE cases, the number of bodies is extremely low (sometimes even 0 or 1), so the search for a significant maximum in the relevant $H$ range cannot be performed even with a reduced box size. The cases for which the fit has been performed with the reduced box size correspond to low numbers of bodies in the region of the expected maximum. The only exception is the family 1726 
for which we adopted a reduced box size to obtain a more detailed $R M A X(H)$, even if the expected maximum is in the range for which we have more than 100 brighter objects. Note that the nominal $R M A X(H)$ might also lead to a reasonable fit.

In conclusion, the main reason for failure to find or difficulty in finding the fit is essentially statistical (too few members in the relevant $H$, or size, range). Similar problems might arise when looking for a fit within very young families, where the relevant $H$ range is in a region where we have no bodies, or where the number of bodies is severely diminished by observational selection effects. Fortunately, the only very young family with a Yarkage is that of (1547) Nele, which is bright enough and located in the inner part of the Main Belt, thus allowing us to find a significant number of bodies with high enough $H$ (around $16 \mathrm{mag}$ ).

\section{RESULTS AND DISCUSSION}

\subsection{The Yarkovsky-YORP comparison: uncertainties and errors}

The present analysis allowed us to identify, for families with a previous age estimate, the significant maxima of $\operatorname{RMAX}(H)$ and, consequently, to obtain a new estimate of the age based on the YORP effect. However, the problem of calibration discussed in Paper I remains unsolved, and thus the age is defined up to a multiplicative constant. A complete and updated model, including YORP, Yarkovsky, collisions and, in some cases, other dynamical mechanisms connected, for instance, to resonances, does not exist, and thus we are bound to refer to the first pioneering attempts by Vokrouhlický et al. (2006). However, we can at least offer a couple of considerations.

(i) In principle, after one YORP cycle, the spin of the bodies should be preferentially oriented close to the normal to the orbit plane, thus maximizing the effectiveness of the Yarkovsky drift. However, as the spin clustering can be partially or totally disrupted by collisions, the maximum clustering might be reached before this time; it is also possible, due to a complex combination of effects, that the maximum clustering will be reached at a later time. This uncertainty has to be added to the basic uncertainty resulting from the calibration of the YORP effect itself, which might also depend on additional parameters, such as size - apart from the well-known $1 / D^{2}$ dependence - or taxonomy, or porosity, etc. Essentially, we have no a priori indication whether the age at which a maximum $R$ at a particular value of $H$ (the 'eye') occurs is larger or smaller than the duration of the YORP cycle corresponding to the same $H$. The results we discuss might, in principle, provide a hint concerning this point, but the combination of several uncertainties, the partial subjectivity of our choice of significant maxima and the intrinsically unknown parameters do not allow us to consider them accurately enough.

(ii) Defining the ansatz in Paper I, we have ignored the fact that the formation of the eye requires both spin clustering and a significant Yarkovsky drift after this clustering. It can introduce an additive offset term in the calibration. We expect that, given the different dependence of YORP and Yarkovsky effects on the size $\left(\propto 1 / D^{2}\right.$ and $\propto 1 / D$, respectively), this term is more important for small objects, and thus for younger families.

It is also worth discussing the errors of the age estimates, and the possible definition of an error bar. The errors involved in the estimate of the age obtained from the Yarkovsky effect (generally not small) have been estimated in Milani et al. (2017). For most families, the nominal value of the age and of the respective error have been obtained as a sort of a weighted average between the IN and OUT estimated ages. The error bars are represented by the horizontal bars in Fig. 8. Error bars are also available for the three cases in which the IN and OUT estimates cannot be reconciled, and we are left with a double age. It is possible that these two ages correspond to two collisional events, as appears to have happened in the above-mentioned case of the family of 4 Vesta. In a way, these cases are not too different from the more numerous cases for which only one wing has been used to obtain an estimate of the age. These families, presumably, often underwent complex postcollision evolutionary paths, involving resonances, for example. Also, in this case, an error bar is available. However, because we have used for the final calibration only the families with two consistent Yarkages, we decided not to represent the corresponding error bars of these two last groups in Fig. 8.

Regarding the error in the definition of the age estimated from the YORP effect, we have essentially two terms. One term, due to the above discussed calibration and similar problems, is essentially unpredictable, and has been ignored in our estimates; the other, which is easier to handle, is connected to the uncertainties/dispersion of the albedo and of the semimajor axis. Let us consider the definition of the YORP-age given in Paper I and recalled by equation (1) (equation 6). Then, if we have a distribution of $A$ with average $A_{\text {mean }}$ and standard deviation $\Delta A$ (often rather large), and a distribution of $a$ with average $a_{\text {mean }}$ and standard deviation $\Delta a$, we obtain

$\frac{\Delta Y_{\text {age }}}{Y_{\text {age }}} \simeq \frac{\Delta A}{A_{\text {mean }}}+2 \frac{\Delta a}{a_{\text {mean }}}+\frac{\Delta \tau_{\mathrm{f}}}{\tau_{\mathrm{f}}}$,

where $\Delta \tau_{\mathrm{f}}$ refers to the uncertainty of the age estimate, which can be obtained from the literature. In principle, this argument can lead us to estimate the uncertainty of the expected value of the maximum $R(H)$, and thus to decide whether the expected and the computed maxima are consistent with each other. However, there are two reasons to follow a different path. First, the errors in albedo and age are usually large, so the linear approach in equation (2) is less reliable. Secondly, and more importantly, we are willing to extend our consideration to families for which a previous age estimate does not exist. Thus, we start from the computed maximum of $R(H)$ to define an age estimate, which we call in the following Yorpage, to be compared, whenever possible, with the existing age estimates. The uncertainty $\Delta$ Yorpage/Yorpage is due only to the spreads in albedo and semimajor axis, and it is given by

$\frac{\Delta \text { Yorpage }}{\text { Yorpage }} \simeq \frac{\Delta A}{A_{\text {mean }}}+2 \frac{\Delta a}{a_{\text {mean }}}$.

The estimates of the error range between 20 and 50 per cent (see also Table 5); the related error bars are represented (vertical bars) in Fig. 8, for the same families for which we have given the Yarkage error bars. Obviously, the same uncertainty applies to the actual age estimates obtained by the YORP effect.

\subsection{Yorpage versus Yarkage plots}

Before a final comparison, which will include a calibration, we can introduce a raw plot, comparing the ages for different groups of objects. Note that we discuss a few peculiar cases in the following. At this stage, we are using all the nominal data as they are. The relevant groups we are going to analyse are the following.

(i) All families: in this sample, we include all the families with any Yarkage estimate, excluding those that have been identified as 
Table 5. A synthetic summary of the results for the good families. We recall the recent changed identifiers for families 293 (now 1521) and 110 (now 363). For every family, we report the Yarkage, the calibrated Yorpage, the estimated error for Yorpage, the total estimated error (see text) and five quality codes (i.e. the absolute maximum, the value of maximum, the difference compared with $\sigma$, the box size reduction code and the order of adopted maximum starting from low $H$; see text for details); in some cases, notes are present, where $1=$ see text.

\begin{tabular}{lcccccccccc}
\hline Corfam & Yarkage & Yorpage & Erryorp & Toterr & Absmax & ValR & Sig & Box & Order & Notes \\
\hline 5 Astraea & 329 & 248 & 0.31 & 0.38 & 0 & 0 & 0 & 0 & 1 & \\
10 Hygiea & 1347 & 1488 & 0.30 & 0.34 & 1 & 1 & 0 & 0 & 1 & \\
24 Themis & 3024 & 3350 & 0.32 & 0.38 & 1 & 0 & 0 & 0 & 1 & \\
31 Euphrosyne & 1225 & 1213 & 0.35 & 0.39 & 0 & 1 & 1 & 1 & 1 & 1 \\
110 Lydia & 238 & 229 & 0.25 & 0.30 & 1 & 1 & 0 & 0 & 1 & \\
158 Koronis & 1746 & 1737 & 0.28 & 0.32 & 1 & 0 & 0 & 1 & 1 & \\
163 Erigone & 224 & 250 & 0.20 & 0.26 & 0 & 0 & 0 & 1 & 1 & \\
221 Eos & 1466 & 1308 & 0.36 & 0.39 & 1 & 0 & 0 & 0 & 3 & 1 \\
293 Brasilia & 230 & 283 & 0.24 & 0.46 & 1 & 1 & 0 & 0 & 1 & \\
302 Clarissa & 50 & 81 & 0.38 & 0.44 & 1 & 0 & 0 & 0 & 1 & \\
396 Aeolia & 96 & 48 & 0.29 & 0.36 & 0 & 0 & 1 & 1 & 1 & \\
569 Misa & 259 & 408 & 0.36 & 0.51 & 1 & 0 & 0 & 0 & 1 & 1 \\
606 Brangane & 46 & 83 & 0.25 & 0.30 & 0 & 0 & 1 & 0 & 1 & \\
668 Dora & 506 & 355 & 0.18 & 0.29 & 0 & 0 & 1 & 0 & 2 & \\
808 Merxia & 329 & 188 & 0.26 & 0.30 & 1 & 0 & 1 & 1 & 1 & \\
845 Naema & 156 & 147 & 0.16 & 0.34 & 0 & 0 & 0 & 0 & 1 & \\
847 Agnia & 753 & 412 & 0.27 & 0.33 & 0 & 0 & 1 & 1 & 1 & \\
1040 Klumpkea & 663 & 607 & 0.50 & 0.55 & 1 & 0 & 0 & 1 & 1 & \\
1128 Astrid & 150 & 107 & 0.20 & 0.25 & 0 & 0 & 0 & 0 & 1 & \\
1303 Luthera & 276 & 461 & 0.20 & 0.30 & 1 & 0 & 1 & 0 & 2 & \\
1547 Nele & 14 & 61 & 0.20 & 0.35 & 1 & 1 & 2 & 0 & 2 & \\
1726 Hoffmeister & 332 & 778 & 0.22 & 0.30 & 0 & 0 & 1 & 1 & 1 & \\
1911 Schubart & 1557 & 1109 & 0.26 & 0.34 & 1 & 1 & 0 & 0 & 2 & 1 \\
3330 Gantrisch & 460 & 436 & 0.22 & 0.35 & 0 & 0 & 0 & 0 & 1 & \\
3815 Konig & 51 & 104 & 0.20 & 0.28 & 1 & 0 & 1 & 0 & 2 & \\
9506 Telramund & 219 & 126 & 0.29 & 0.37 & 1 & 1 & 1 & 0 & 1 & 1 \\
18405 1993FY12 & 83 & 81 & 0.22 & 0.28 & 0 & 0 & 0 & 0 & 1 & \\
\hline
\end{tabular}

OUTRANGE. Those with two consistent ages are included twice; this does not affect the figure, but it is relevant for the computation of linear regression (see below).

(ii) Families with a good Yarkage: a subset of the previous sample including only those with two consistent Yarkage estimates.

(iii) In a recent paper, Milani et al. (2018) have explicitly divided the families between 'fragmentation' and 'cratering'; we define a sample with only the cratering families. Note that, for the purpose of the present work, we are not taking into account the possible problems connected with this classification (i.e. transition or ambiguous cases).

(iv) As above, we define a sample with only the fragmentation families.

In Fig. 7, we represent these four samples in a single plot. We have plotted the age obtained with the YORP analysis (hereafter Yorpage, to be distinguished from the definition of $Y_{\text {age }}$ used above and in Paper I) versus the age estimated on the basis of the Yarkovsky effect (Yarkage). Obviously, the symbols that refer to the fragmentation and cratering families are superimposed on those corresponding to the 'all' and 'good' samples. Also, for cratering or fragmentation samples, the objects with two consistent ages are reported twice. In the figures, we also show the linear regression plots that refer to the samples. As is easy to see, they indicate in all cases a small negative offset (a few tens of Myr) and a coefficient of proportionality $\alpha$, which is typically moderately larger than unity. Indeed, the proximity of $\alpha$ to unity is good and significant news, and not at all predictable. Also, the offset can have a physical significance. As we have discussed in the previous subsection, our ansatz - the Yorpage is equal to the duration of a YORP cycle for the magnitude where the maximum of $\operatorname{RMAX}(H)$ appears - was neglecting the time required by the Yarkovsky effect to move the objects in the semimajor axis. This neglected time is more relevant for the smaller objects, because of the different size dependence for the YORP and Yarkovsky effect; thus, the young families (for which the relevant maximum corresponds to small objects) can seem younger when their age is computed with the YORP method. Note, however, that the estimated offset resulting from the regression relation can be a dominant effect for very young families; for these objects, the calibration, which we discuss below, can overcorrect the estimated age (see the discussion in the following). The coefficients that refer to the 'all' and 'good' samples amount to about 1.2; the coefficient corresponding to fragmentation families is slightly smaller, while it becomes steeper for cratering families. There is no obvious explanation for this difference, and we have to remark that there are few cratering families, so the difference might be due to statistical fluctuations. However, further analysis might be useful, with the perspective of a fraction of cratering families also increasing in the future (Milani et al. 2018).

Finally, we introduce error bars and the calibration issue in Fig. 8. We have made a choice that we consider to be reasonable: because the families with only one Yarkovsky age and those with two inconsistent ages are expected to have undergone a complex collisional (multiple relevant collisions) or dynamical (truncating resonances, and so on) evolution, we decided to consider for the final calibration only those (27) with two ages (i.e. those defined as good). Thus, the calibration consists of dividing the YORP computed age 


\section{YORP vs YARK}

Comparison of various samples

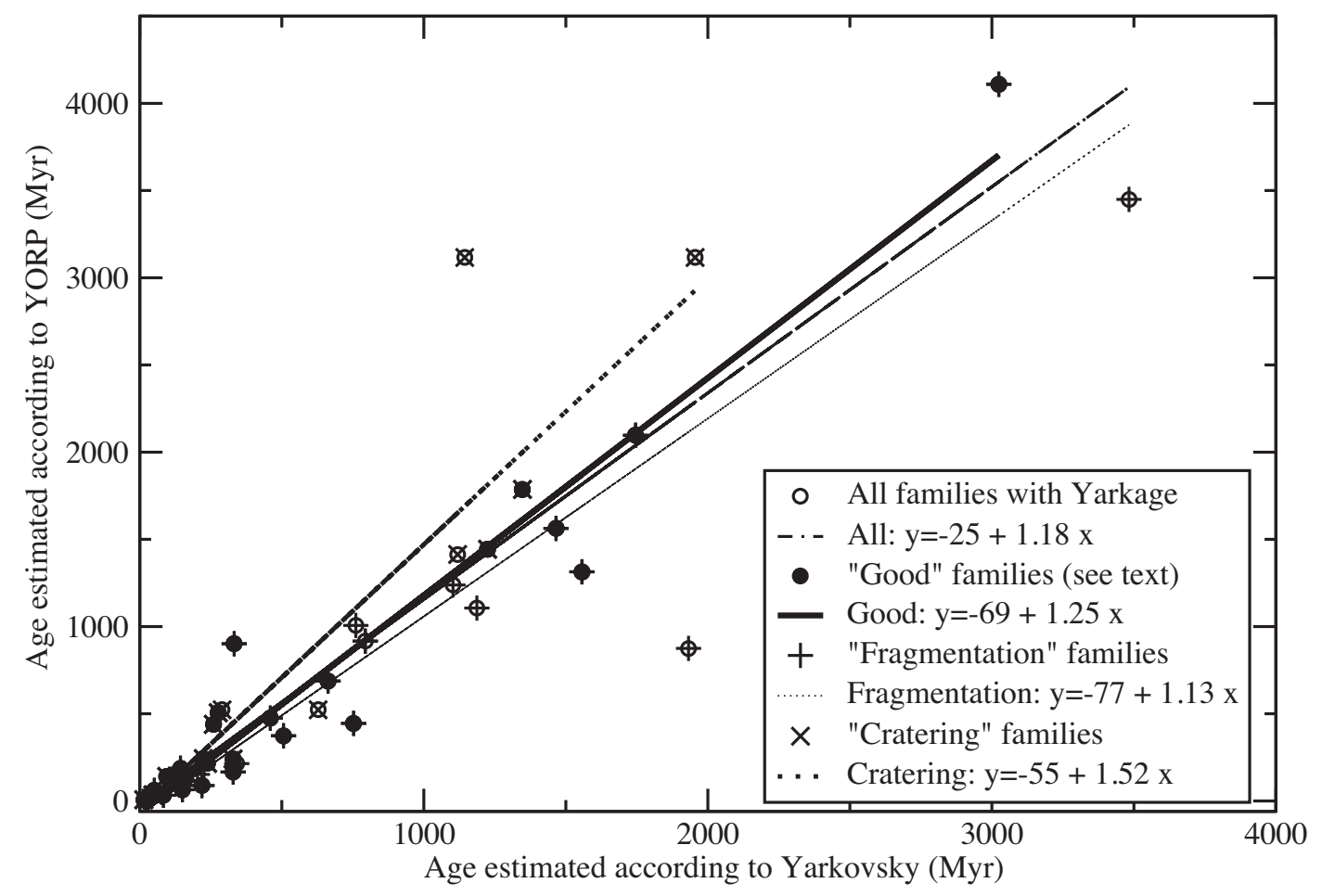

Figure 7. The uncalibrated Yarkovsky and YORP ages for the samples described in the text. Linear regression plots are included. The symbols are explained in the figure legend.

by a factor $\alpha=1.2471$ and adding a constant offset of $55.35 \mathrm{Myr}=$ $69 \mathrm{Myr} / \alpha$. The calibrated Yorpage estimates are plotted against the Yarkage estimates in Fig. 8, where we include also the error bars corresponding to the uncertainty in the Yarkage (horizontal bars) and to the internal error of the Yorpage (vertical bars). We also show the families with one (or two inconsistent) ages, without error bars, with the same calibration. As is obvious, the regression line of the good families has an angular coefficient equal to unity, and no offset; with the same calibration, as expected according to the results previously discussed, the regression for all families has an angular coefficient slightly smaller than unity (about 0.92 ) and a moderate positive offset. We decided not to represent the error bars of these additional families (which are, typically, of a similar size to the others), to ensure the good readability of the graph.

Regarding the quality of the fit, we see that, at least for the good families, the error bars cross the fit line in most cases. There are a few exceptions, however.

Taking into account that the typical YORP and Yarkovsky errors are of a few tenths, that they are at least in part independent from each other and that the $2 \sigma$ acceptance level is reasonable, we have decided to consider the results good enough whenever the Yarkovsky and YORP estimates are within a factor of 2. We have only three cases exceeding this limit. We will improve the analysis in the following, when introducing the total error estimate. The analysis will show that we are even conservative taking these three cases as troublesome. One of these three cases is that of family 1726, whose anomalous behaviour might be connected to the peculiar shape properties discussed by Novaković et al. (2015). The other two cases refer to two young families (i.e. 1547 and $3815)$; in these two cases, the obvious explanation is connected to the constant offset term in the calibration, which dominates the final age estimates for these young families (see also the discussion below).

The results are not seriously affected by these poorly fitted families. In order to verify this statement, we have tried to eliminate all the families not within the above-mentioned factor of 2 in age. The results are plotted in Fig. 9.

The overall fit is not significantly altered; the regression line for the good families has now a coefficient of about 1.01, and the coefficient for all families remains essentially the same; only the constant offset terms are a little different. Consequently, our results are sufficiently robust and reliable.

In Table 5, we represent the overall properties of the good families, relevant for the plot shown in Fig. 8. We have also introduced an estimate of the total standard error Toterr, for a more precise evaluation of the quality of the fit between Yorpage and Yarkage. It is a rough estimate, as we are not analysing the possible interdependences of the errors, and other possible problems. Essentially, we assume that

Toterr $=\sqrt{\left(\frac{\Delta \text { Yarkage }}{\text { Yarkage }}\right)^{2}+\left(\frac{\Delta \text { Yorpage }}{\text { Yorpage }}\right)^{2}}$,

where, obviously, $\Delta X$ is the error related to the quantity $X$. 


\section{TYORP vs TYARK}

Calibrated TYORP (0.802) values

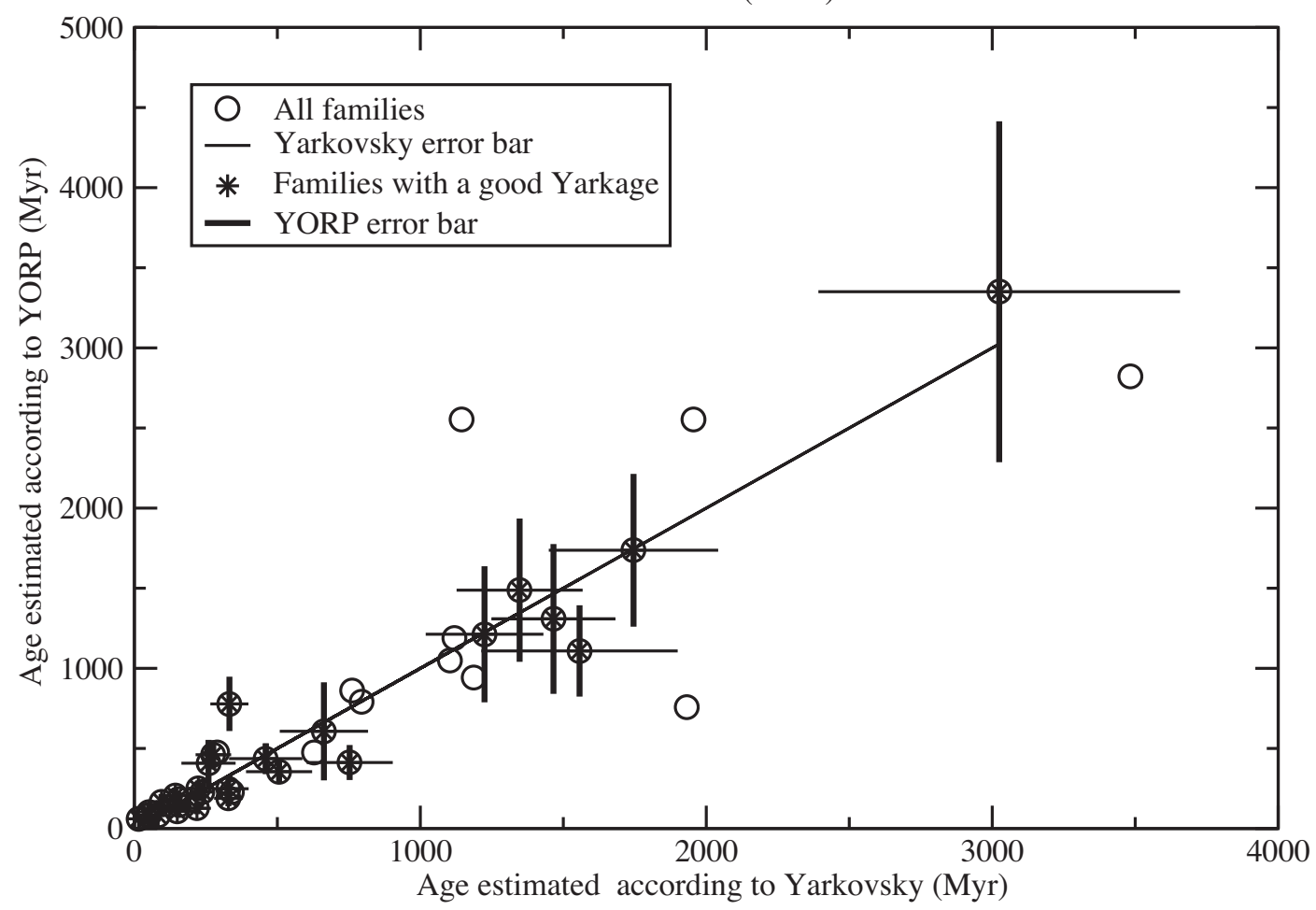

Figure 8. The families after the calibration of the Yorpage. In abscissa, the Yarkage is plotted. In ordinate, we represent the calibrated Yorpage (see text). The line corresponds to the best linear fit, including also a constant offset. The horizontal error bars refer to the estimated error bar for Yarkage, while the vertical error bars refer to the Yorpage uncertainty due to the dispersion of the albedo and semimajor axis within the family.

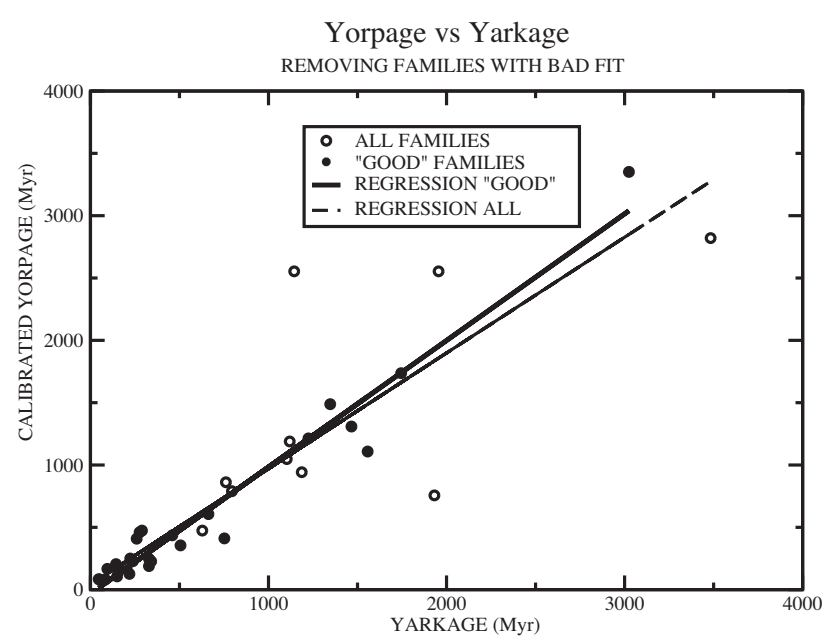

Figure 9. The families without those for which the calibrated Yorpage and Yarkage were different by more than a factor of 2; we adopted the same normalization as in Fig. 8. We also show new regression lines.

We include in the table several quality codes, which answer simple, but relevant, questions, as follows.

(i) Is the adopted maximum one that corresponds to the absolute maximum of $R M A X(H)$ ? YES $=0$; NO $=1$. (ii) Does the adopted maximum $R M A X$ exceed unity? YES $=0$; $\mathrm{NO}=1$

(iii) Is the adopted maximum within $1 \sigma(=0)$ or $2 \sigma(=1)$ with respect to the Yorpage $(\mathrm{NO}=2)$ ? Note that we have computed the standard error multiplying the larger between Yarkage and Yorpage with the relative total error. This procedure leads us to obtain a fit below $2 \sigma$ for all the families, except 1547 . It might be a little optimistic, but not unreasonable. Note that as Yorpage we have used the calibrated value.

(iv) Has the adopted maximum been obtained with a reduced box size? $\mathrm{YES}=1 ; \mathrm{NO}=0$.

(v) Are there significant maxima at smaller values of $H$, compared to the adopted one: $\mathrm{NO}=1$, one significant maximum $=$ 2 , two significant maxima $=3$. According to the discussion in the previous section of what we do understand theoretically, the maxima corresponding to the lowest or to the second lowest $H$ value are usually the most significant.

Finally, note that in the case of family 302, the location of the YORP-eye in $a$ is almost exactly coincident with a relevant resonance. It is the most striking case (but not the only one) of interferences between the features due to the physical evolution and those due to dynamical processes. In this case, the observed 'eye' is partially or totally due to the resonance, and the claimed 'good' result might even be an artefact. In other case, the interference can be disruptive. We have kept this family in our list, as the purpose of the paper is essentially of a statistical nature. However, again we warn the reader that if results concerning individual 
FAMILY 31 Euphrosyne (nominal)

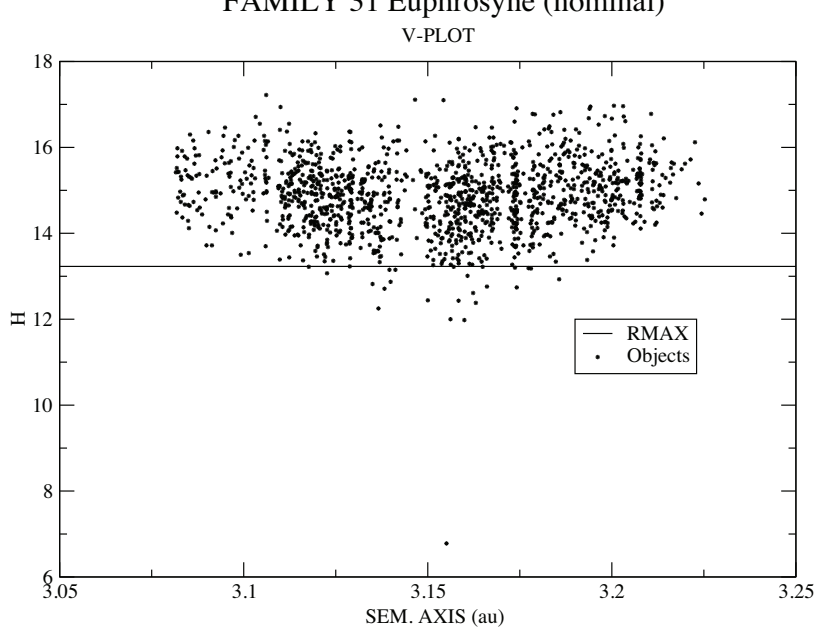

Figure 10. In the V-plot family of (31) Euphrosyne, we represent, with a horizontal line, the $H$ value at which we find the significant maximum of $R M A X(H)$ that we adopted to estimate the Yorpage.

cases are desired, they require a thorough and detailed analysis case-by-case.

\subsection{Particular cases: families 31, 87, 569 and 9506}

The results presented in the previous subsection are statistical; the families are analysed with a general algorithm, ignoring their peculiarities. Even if the overall results, which are of a statistical nature, are robust and do not depend on these peculiarities, it is worth discussing some particular cases, to which Milani et al. (2018) devoted a detailed discussion. The families we are going to consider are of (31) Euphrosyne, (87) Sylvia, (569) Misa and (179) Klytaemnestra (or, as discussed below, of (9506) Telramund).

\subsection{Euphrosyne}

According to the discussion presented in Milani et al. (2018), this dynamical family has a very complex structure, and might even be formed by three collisional families, one corresponding to the wings used for the Yarkage estimate, and two others, probably much younger and compact. We are not going to analyse this or alternative possibilities, nor the overall role of the resonant regions, studied by Machuca \& Carruba (2012). Our purpose is only to discuss why our age estimate (performed with the use of the complete family, including all bodies) is in very good agreement with the Yarkage.

In Fig. 10, in the V-plot of the family, we plot a line corresponding to the $H$ value for which we have identified the relevant RMAX. As can be seen from the figure, at this level the dominant role of the resonant region, discussed by Milani et al. (2018), is not yet completely effective, and the structure seems to exhibit a wider empty region (unfortunately, the statistics is poor, as large bodies are involved). Thus, it is not surprising that we have found a maximum with a very good fit to that expected for the complete family, because the large bodies precisely shape the wings, which are significant to compute the Yarkage.

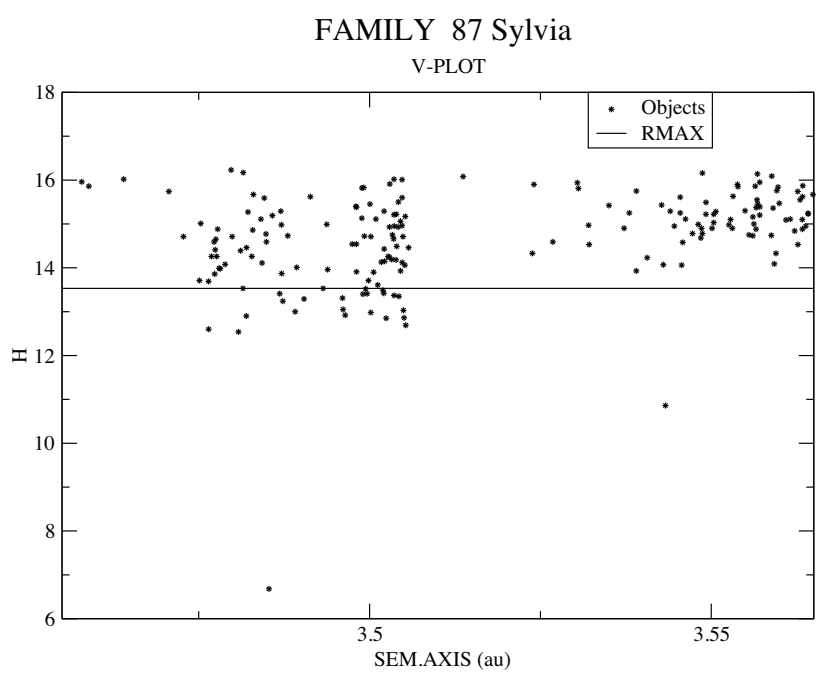

Figure 11. In the V-plot of family of (87) Sylvia, we represent, with a horizontal line, the $H$ value at which we find the significant maximum of $R M A X(H)$ that can be adopted to estimate the Yorpage.

\subsection{Sylvia}

The case of family of (87) Sylvia is not completely different as, in this case, the structure of the V-plot is also dominated by a resonant region. Also, the potentially significant $R M A X$ is at a low $H$ value, for which we have few bodies, all on one side of the resonance. Thus, we are unable to identify possible YORP footprints not making part of the empty region caused by the resonance. Fig. 11 shows this controversial case.

\subsection{Misa}

According to Milani et al. (2018), the family of (569) Misa contains another subfamily, that of (15124), 2000 EZ39. The separation of the two families is difficult, as the largest remnants of both families have a very similar semimajor axis. However, it is possible to define the family 15124, with about 500 members, and to obtain an estimate of the age, slightly above $100 \mathrm{Myr}$. We have not included the family 15124 in the statistical analysis discussed above, but we have run our code and obtained $R M A X(H)$. The main maximum of the function corresponds, after calibration, to an age of about $200 \mathrm{Myr}$, with a reasonable agreement with the Yarkage, taking into account all the uncertainties concerning the identification of the family. The V-plot of the family is reported in Fig. 12, together with a line showing the maximum of $R M A X$.

\subsection{Telramund}

According to the discussion in Milani et al. (2018), the family of (179) Klytaemnestra might be an artefact of the clustering method. In reality, the real and relevant family should be a subset, for which the leading body is 9506 Telramund. In the present paper, we have taken this analysis for granted, and we have used the family 9506 as the significant family.

\subsection{The un-Yarkaged families}

The 16 families listed in Table 3 do not have any age estimate based on the Yarkovsky method. We have included them in our computations, obtaining for each of them a $\operatorname{RMAX}(H)$ function. We 
FAMILY 15124 2000EZ39

V-PLOT

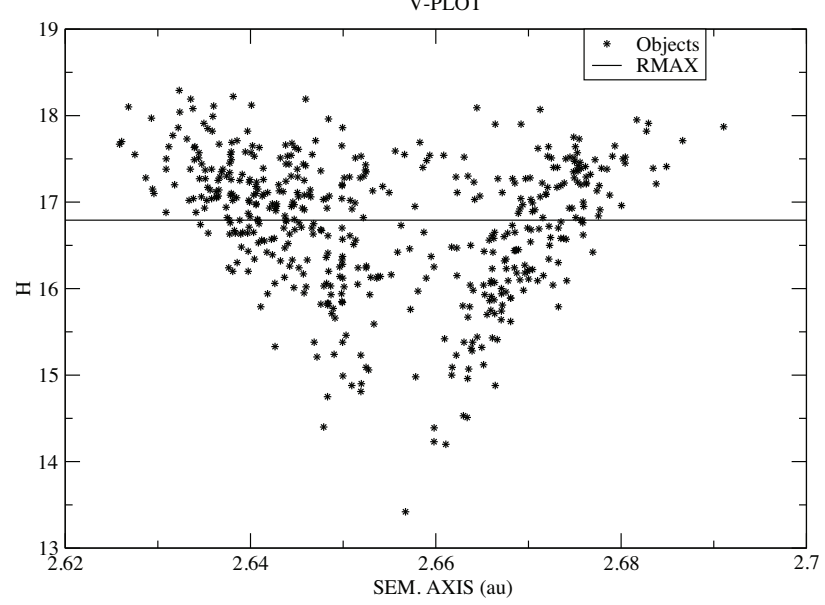

Figure 12. In the V-plot of family 15124, we represent, with a horizontal line, the $H$ value at which we find the significant maximum of $R M A X(H)$ that we adopted to estimate the Yorpage.

used the same approach as for other families to detect the significant maxima. However, in the present case, we have no independent indication, so we have no criterion for a choice among them. Thus, we obtain a list of potential ages computed according to the YORPbased method; the list is slightly subjective, but reliable in principle, as we have used the same method that has given good results for the other families. Unfortunately, we have no way to discriminate among the multiple maxima, whenever they are present, and our outcome is only a list of possible ages, with the hope to being able to confirm or falsify them with a forthcoming analysis, caseby-case, based on additional information. The goal of the present paper is limited to obtaining this list. Anyway, we are prepared for a comparison with the Yarkovsky-based ages, which will be available in the future, or which can be estimated somehow from the data already available, even with a worse precision than usual. It is thus worth converting the computed Yorpage to what could be expected, were the Yarkage obtainable. In the previous discussion, we have found a correction factor and a fixed offset required to obtain, on average, coincident YORP and Yarkovsky ages. The overall equation is of the form

Yorpage $=A+B$ Yarkage,

where $A$ and $B$ are constant quantities. Thus,

Yarkage $=$ Yorpage $/ B-A / B=\operatorname{Corr}_{Y \text { age }}+55.35$,

where the quantities are, as usual, in Myr and Corr $_{Y a g e}$ is the corrected value (by a factor $A=0.802$ ) of the age used to obtain the plot in Fig. 8.

Moreover, we have a few weak indications from the Yarkovskybased analysis, and a pair of age estimates obtained with different methods: (490) Veritas (Knežević, Tsiganis \& Varvoglis 2006; Carruba et al. 2018) and (778) Theobalda (Novaković 2010). Thus, we give a list of potential ages, with a synthetic conclusion, and a few notes about the possibility of accepting these estimates as reliable. The list of these families and of their suggested ages is presented in Table 6.

In Table 6, please note the following.

(i) According to the above quoted estimates present in the literature, the families 490 and 778 are very young, and thus the suggestions presented in the table have to be considered not meaningful.

(ii) Five families of the sample have Mem $\leq 120$ (see Table 3). These families do not have any Yarkage as the number of their members is too low to allow a significant statistical analysis; probably the same considerations apply also to our Yorpage estimates. Because future updates of the data base and thus the growth of family memberships are expected, we decided to reserve these cases for future reference.

(iii) For the other families, age estimates might be available in the near future. While preparing this paper, a new Yarkovsky-based age determination was added (for family 87; see Table 2). The estimated Yorpage we obtained before this determination was in good agreement.

\subsection{The problem of young and very young families}

In the previous subsections, we have pointed out that, in our analysis, there are some problems when discussing the properties of young families. There are several reasons for this.

(i) An obvious problem concerns the calibration we performed to compare Yorpage with Yarkage. Our calibration entails a fixed offset of about $50 \mathrm{Myr}$, so the minimum expected calibrated age is equal to this offset (for a nominal zero un-calibrated Yorpage). In this way, we are, by definition, unable to fit ages below $50 \mathrm{Myr}$, and we are presumably overestimating the ages of families slightly older than this limit. In physical terms, the reason is simple. The age at which the 'eye' should appear is the sum of the time required to align the rotation axes, due to the YORP, plus the time to change the semimajor axes, due to the Yarkovsky effect, large enough to be observed in the V-plot. As YORP depends on the size $D$ as $1 / D^{2}$ while the Yarkovsky effect depends only on $1 / D$, this additional term is negligible for old families, but not for young families, with the relevant sizes (or magnitudes $H$ ) to find the 'eye' being smaller. Thus, the presence of this fixed offset is, as for a first approximation, physically grounded. However, it is true that the time required for a sufficient Yarkovsky-driven mobility is also shorter for the young families, as the objects relevant for the detection of the evolution of the V-plot are smaller. In principle, one might improve the fit with a size-dependent offset. We decided not to do so for simplicity, also taking into account that, in our sample, the families for which this correction might be significant are very few. However, it does count, as can be seen with the help of Fig. 13. In the figure, we represent the nominal RMAX, which corresponds, before the calibration, to a nominal age of about $7 \mathrm{Myr}$, in striking - but casual - agreement with the new age estimate, based on the convergence of the secular angle, by Carruba et al. (2018). We might also, by removing the control on the observational selection effects (see above), find another maximum, at about $17.8 \mathrm{mag}$, with a nominal age - before calibration - of about 2.5 Myr.

(ii) Another problem concerns the values of $H$ (or size) for which we should find significant footprints of the YORP/Yarkovsky evolution of the V-plot. They are, for families with ages of the order of a few Myr, clearly beyond the completeness limit; thus, we are working on a subsample of the real members. This problem is serious, but we can expect future observations to help to mitigate it. At present, the best age estimates for these families come from dynamical computations, such as the BIM discussed by Carruba et al. (2018).

(iii) Finally, some families are so young that no significant YORP/Yarkovsky evolution had time to occur: these are the so- 
Table 6. For the un-Yarkaged families, we give the number of significant maxima and the corresponding possible age range(s) (in Myr) suggested by the YORP analysis and calibrated to obtain the expected Yarkage(s). We give also a synthetic estimate.

\begin{tabular}{lccc}
\hline Corfam & $N_{\max }$ & Expected Yarkage(s) (in Myr) & Synthetic estimate \\
\hline 96 Aegle & 3 & $195-244,404-525,777-1028$ & $\simeq 100-1000 \mathrm{Myr}$ \\
148 Gallia & 1 & $91-119$ & $\simeq 100 \mathrm{Myr}$ \\
298 Baptistina & 2 & $56-58,72-90$ & $<100 \mathrm{Myr}$ \\
410 Chloris & 1 & $161-264$ & $\simeq 200 \mathrm{Myr}$ \\
490 Veritas & 3 & $117-167,224-362,821-1447$ & $\simeq 200 \mathrm{Myr}$ or $1 \mathrm{Gyr}$ \\
778 Theobalda & 2 & $102-143,265-451$ & $>100 \mathrm{Myr}$ \\
883 Matterania & 2 & $69-86,104-163$ & $\simeq 100 \mathrm{Myr}$ \\
1118 Hanskya & 2 & $146-190,2430-3589$ & $\simeq 150 \mathrm{Myr}$ or $3 \mathrm{Gyr}$ \\
1222 Tina & 2 & $68-75,96-116$ & $\simeq 100 \mathrm{Myr}$ \\
1298 Nocturna & 2 & $172-263,431-1017$ & $\simeq 100 \mathrm{or} 700 \mathrm{Myr}$ \\
1338 Duponta & 3 & $105-125,17-92,97-147$ & $\simeq 150 \mathrm{or} 600 \mathrm{Myr}$ \\
2782 Leonidas & 3 & $59-68,66-94,89-173$ & $\simeq 100 \mathrm{Myr}$ \\
12739 1192DY7 & $134-176$ & $\simeq 150 \mathrm{Myr}$ \\
13314 1998RH71 & 3 & $66-78,87-121$ & $\simeq 100 \mathrm{Myr}$ \\
18466 1995SU37 & 1 & $81-107,120-182$ & $\simeq 100 \mathrm{Myr}$ \\
31811 1999NA41 & 2 & &
\end{tabular}

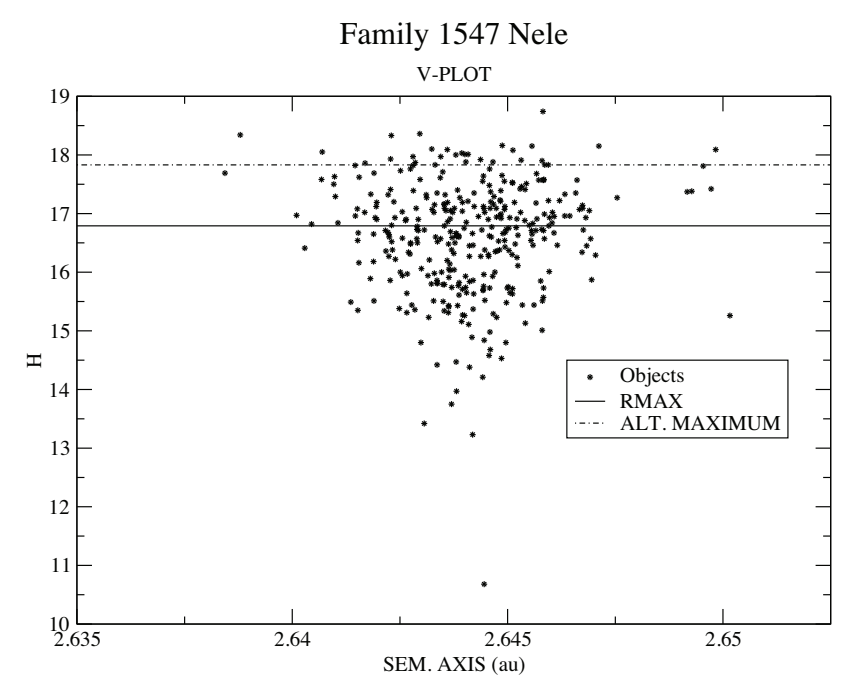

Figure 13. In the V-plot of family 1547 , we represent, with a horizontal line, the $H$ value at which we find the significant maximum of $R M A X(H)$ that we adopted to estimate the Yorpage, and a possible alternative maximum (see text).

called ‘very young' families (Rosaev \& Plávalová 2018). These families are presumably destined to remain unavailable for our analysis, now and in the future.

\section{CONCLUSIONS AND OPEN PROBLEMS}

In this paper, we have finalized the method and the ideas presented in Paper I. The search of the YORP-eye has proven fruitful, and allows us to obtain age estimates, in most cases in good agreement with the estimates obtained with the analysis based on the Yarkovsky effect. Some families are unfit for the YORP analysis: typically old families, for which the 'eye' should be located at small values of $H$ where few bodies are present, or families for which there is a large gap in size among the one or few large fragments and the others. The algorithm is not able to resolve the structure of the function $R M A X$ in the $H$ region for which it is expected to be significant.
In general, however, the method works well, and the frequent fair agreement between Yorpage and Yarkage supports the reliability of both estimates, and the assumptions behind them.

We have also introduced an estimate of the error of Yorpage, depending mainly on the dispersion of albedos. However, we have to remark again that the results suffer from other, partially unpredictable, uncertainties. Apart from the already discussed problem of calibration, which, however, the present direct comparison between Yorpage and Yarkage might help to resolve, we have other problems (interlopers, asymmetric structure of the family, different parameters working for different taxonomic types or even individual families, mixing of other dynamical effects, etc.). Thus, we have to emphasize again that the outcomes from the present analysis provide only an indication, meaningful but not necessarily accurate enough, and need to be supported by other independent evidence.

Thus, the values we obtain for the un-Yarkaged families are no more than a starting point for a thorough case-by-case analysis.

Finally, we wish to remark, as already pointed out by Paolicchi et al. (2017), that a theoretical model, putting together original properties, Yarkovsky and YORP, dynamics and - last but not the least - collisions after the formation of the family, is urgently needed, to update and improve the pioneering approach by Vokrouhlický et al. (2006). This project might also allow a better analysis concerning the young families.

A possible future analysis might also be devoted to the symmetry properties of the families, including the analysis of the third (skewness) and fourth (kurtosis) momenta in the distributions of $a, e$ and $I$. The kurtosis analysis as a tool to understand the properties of asteroid families has been already introduced by Carruba \& Nesvorný (2016), and has been devoted to the symmetry properties concerning the inclination $I$ (or the velocity component $v_{w}$ ). This provided useful indications about a few families, which have been studied in subsequent papers, also taking into account the effects of secular resonances with Ceres. A three-dimensional analysis might be helpful to identify the combination of evolutionary processes, thus enabling us to move from a statistical study to an understanding of individual families, according to the ideas discussed by Milani et al. (2018) 


\section{ACKNOWLEDGEMENTS}

Sadly, Andrea Milani passed away on 28 November 2018. The other authors wish to dedicate this paper to Andrea, acknowledging his outstanding contribution to the science of Celestial Mechanics and Minor Bodies. ZK acknowledges support from the Serbian Academy of Sciences and Arts via project F187, and from the Ministry of Education, Science and Technological Development of Serbia through the project 176011 . PP acknowledges funding by University of Pisa and INFN/TAsP. We are grateful to the referee, V. Carruba, for useful suggestions.

\section{REFERENCES}

Bottke W. F. et al., 2015, Icarus, 247, 191

Bottke W. F., Vokrouhlický D., Rubincam D. P., Brož M., 2002, in Bottke W. F., Jr, Cellino A., Paolicchi P., Binzel R. P., eds, Asteroids III. Univ. of Arizona Press, Tucson, p. 395

Bottke W. F., Vokrouhlický D., Rubincam D. P., Nesvorný D., 2006, Ann. Rev. Earth Planet. Sci., 34, 157

Carruba V., D. Nesvorný, 2016, MNRAS, 457, 1332

Carruba V., De Oliveira E. R., RodriguesB., Requena I., 2018, MNRAS, 479,4815

Chesley S. R. et al., 2003, Science, 302, 1739

Farinella P., Vokrouhlický D., 1999, Science, 283, 1507

Farinella P., Vokrouhlický D., Hartmann W. K., 1998, Icarus, 132, 378

Knežević Z., Milani A., 2003, A\&A, 403, 1165

Knežević Z., Tsiganis K., Varvoglis H., 2006, Pub. Astron. Obs. Belgrade, 80,161

Machuca J. F., Carruba V., 2012, MNRAS, 420, 1779
Micheli M., Paolicchi P., 2008, A\&A, 490, 387

Milani A., Cellino A., Knežević Z., Novaković B., Spoto F., Paolicchi P., 2014, Icarus, 239, 46

Milani A., Spoto F., Knežević Z., Novaković B., Tsirvoulis G., 2016, in Chesley S., Farnocchia D., Jedicke R., Morbidelli A., eds, Proc. IAU Symp. Vol. 318, Asteroids: New Observations, New Models. Cambridge Univ. Press, Cambridge, p. 28

Milani A., Knežević Z., Spoto F., Cellino A., Novaković B., Tsirvoulis G., 2017, Icarus, 288, 240

Milani A., Knežević Z., Spoto F., Paolicchi P., 2018, preprint (arXiv:1812 .07535)

Morbidelli A., Nesvorný D., Bottke W. F., Michel P., Vokrouhlický D., Tanga P., 2003, Icarus, 162, 328

Nesvorný D., Vokrouhlický D., 2008, AJ, 136, 291

Novaković B., 2010, MNRAS, 407, 1477

Novaković B., Maurel C., Tsirvoulis G., Knežević Z., Radovic V., 2015, Dynamical portrait of the Hoffmeister asteroid family, IAU General Assembly, Meeting 29, \#2251337

Paolicchi P., Knežević Z., 2016, Icarus, 274, 314 (Paper I)

Paolicchi P., Knežević Z., Spoto F., Milani A., Cellino A., 2017, EPJP, 132, 307

Rosaev A., Plávalová E., 2018, Icarus, 304, 135

Spoto F., Milani A., Knežević Z., 2015, Icarus, 257, 275

Vokrouhlický D., Capek D., 2002, Icarus, 159, 449

Vokrouhlický D., Brož M., Bottke W. F., Nesvorný D., Morbidelli A., 2006, Icarus, 182,118

Vokrouhlický D., Bottke W. F., Chesley S. R., Scheeres D. J., Statler T. S., 2015, in Michel P., DeMeo F. E., Bottke W. F., eds, Asteroids IV. University of Arizona, Tucson, p. 509

This paper has been typeset from a $\mathrm{T}_{\mathrm{E}} \mathrm{X} / \mathrm{L} \mathrm{T} \mathrm{E} X$ file prepared by the author. 